\title{
TRAINING IN ASTRONOMY
}

\author{
M. GERBALDI \\ CNRS Institut d'Astrophysique and Université de Paris Sud XI \\ 98bis Bd Arago, 75014 Paris, France, \\ e-mail: gerbaldi@iap.fr
}

\section{Introduction}

Astronomy offers a unique opportunity for promoting the science teaching in its present crisis. Astronomy can be introduced at various levels and become the medium by which both primary science education and public understanding of science are stimulated.

At the University level, astronomy can be introduced in the curricula of university colleges and be a subject for M.Sc. and Ph.D. degrees. Astronomy, can give students the opportunity to work scientifically from observations and known physical laws in order to derive knowledge in another field of science. Astronomy can be taught with less formalism and more experimentation, giving students a feel for the link between a phenomenon and its theoretical representation, and how and why a given observation can be represented by different theoretical models.

At School level, astronomy can be introduced in either junior or senior high schools. The objective is not only to teach an extra subject but to widen students' knowledge of science and to show them how to work scientifically. Astronomy can help fight math phobia. But astronomy can be taught efficiently at school level only if teachers have enough specific astronomical knowledge to dare to deal with the topic. So teacher training, before or during service, is extremely important in this context. This poses problems in developing countries with very few astronomers, or in large sparsely populated countries.

\section{Teaching of Astronomy at the University}

At the University astronomy can be taught at 3 levels :

For undergraduate students, to broaden their fields of view in physics

For the training of schoolteachers

To train astronomers at MSc or PhD level.

Starting with the last point: very few developing countries offer MSc or PhD degrees in astronomy, but students may obtain such diplomas in neighboring countries, if grants are available. For example, in South America, students from Peru, Uruguay, etc are undertaking their graduate studies in Argentina, and now Brazil and Mexico. Also, students from Indonesia, for example, go to Japan. Regional relationships between countries are now developing in this context.

Undergraduate students can often study locally and the national reports published by IAU Commission 46 (see its home page on the Web) show that a large number of developing contries offer teaching at this level. How do we have courses that are not exclusively in theoretical astronomy? How do we give students a feel for the observational part of astronomy? There are two aspects of access to the observational domain of astronomy to be developed : observations with a telescope, and analysis of data from the data bases and aechives of any ground-based telescope or space observatory.

Many places have modest telescopes that can be used very efficiently to teach the planning of observations. If these telescopes are equipped with a CCD or a simple photometer, they can be used to teach the planning and conduct of observations and to give an understanding of the main sources of errors. Very often, older books give more detailed guidelines for making such observations than do the most recent ones, which emphasize technological aspects. 
We have found, in developing countries, that PCs are available, but without software that would permit proper data analysis. There is software such as MIDAS or IRAF, developed by the astronomical community for research, and available free of charge; but their use in developing countries is hampered by the absence of any "system manager" who knows how to implement them. Such drawbacks must be taken into account in any project.

Nevertheless the situation is far from desperate and at undergraduate level very valuable work can be done with data extracted from a data base, even without an Internet line or with one too slow to extract data remotely. It should be remembered that data from several space experiments are available on CD-Rom. Take the example of the HIPPARCOS Input Catalogue : one has been produced to be used with personal computer and the necessary software is also on a CD-Rom. A similar CD-Rom will be produced very soon, with the data from this experiment. It should be emphasized that such a CD-Rom contains valuable data which permits good training for undergraduate students with real and modern data.

\section{Teaching of Astronomy at School}

Once more it is striking to discover from the Triennial Reports of Commission 46 that many countries have some astronomy in their school curricula, but not many have teacher training. We can predict the consequence: astronomy teaching will not have a long lifetime where there is no teacher training.

In no country can in-service teachers training be introduced either quickly or on a large scale: it must be a slow but continuous process. The process has been shown to be extremely efficient when a small but motivated group of teachers provides the impulse. In fact, in any country a network of trained school teachers can be created, starting from a small nucleus of people. These teachers will then act at the local level as instructors for their colleagues.

In all countries, teachers need a theoretical knowledge of astronomy but, because of the wide differences in their backgrounds, it is best to lead to the theoretical knowledge through practical exercises; this will also increase teachers' familiarity with observation and experiment, and they will transfer that to their pupils.

Astronomy training for schoolteachers also provides oppportunities for them to meet and to work together, to talk and to exchange experiences, overcoming the barriers between disciplines. In-service training should be organized to produce instrcution manuals. Simple manuals developed by the teacher in the classroom are preferable to ready-to-use packages. All manuals should be very low-cost but of very good educational quality, so they must have been thoroughly tested on pupils by several teachers. The manuals should be easy to use and each practical activity in them should have modest and well-defined objectives. When exchanging manuals between countries, the cultural aspect is important: a given activity may need to be presented in a differently in another country.

\section{References}

The Centre de Données Astronomiques de Strasbourg (Strasbourg Astronomical Data Center) can be reached at the following addresses :

http://cdsweb.u-strasbg.fr

$\mathrm{ftp}: / /$ cdsarc.u-strasbg.fr/pub/cats

Papers can be found in:

5th International Conference Teaching Astronomy

Published by the Universitat Politecnica de Catalunya, Institut de Ciènces de l'Educació

see for example: Astronomy Practical Activities and the New French Programmes, by L. Bottinelli, M. Gerbaldi and L. Gouguenheim.

IAU Colloquium 16\%: New Trends in Astronomy Teaching (1996) to be published 